\author{
Anna Małgorzewicz \\ ORCID 0000-0001-5366-3957 \\ Universität Wrocław, Wrocław
}

DOI: $10.19195 / 0435-5865.144 .14$

\title{
Universitäre postgraduale Translatorenausbildung - strategische Konzepte, Praxiserfahrungen und Ergebnisse
}

\begin{abstract}
s
Der Beitrag setzt sich mit den Fragen der universitären Translatorenausbildung auseinander, welche im Rahmen der postgradualen Studiengänge an polnischen Hochschulen realisiert wird. Am Beispiel eines postgradualen Studiengangs für Übersetzer und Dolmetscher wird der Entstehungsprozess des Lehrprogramms gezeigt, dessen Grundlage das Definieren der zu erzielenden Kompetenzen, Fähigund Fertigkeiten bildet. Es wird auf Ermittlungen einer empirischen Untersuchung hingewiesen, die es möglich machte, Desiderate für die universitäre Translatorenausbildung zu formulieren. Die Autorin bespricht im Weiteren Komponenten der didaktischen Strategie, welche in einem dynamischen und determinierenden Verhältnis zueinander stehen und gerade in dieser Determiniertheit zum Fokus der translationsdidaktischen Betrachtungen gerückt werden. Die geschilderten Annahmen und vertretenen konzeptionellen Ansätze werden in ihrer praktischen Realisierung geschildert.
\end{abstract}

Schlüsselwörter: postgraduale Translatorenausbildung, strategische Translationskompetenz, Lehrprogramm für Translatorenausbildung, didaktische Strategie, Translationskompetenz

\section{Postgraduate courses for translators at university level - strategic concepts, practical experience and results}

The paper discusses the subject of postgraduate courses for translators offered at Polish universities. One example of such postgraduate courses for translators and interpreters illustrates the process of development of curriculum in which the desired competences, abilities and skills are a determining factor. The paper refers also to the results of empirical research which made it possible to formulate desiderata for courses for translators at university level. Moreover, the author examines the components of didactic strategy that are in a dynamic and determined relation to one another. The determined character of this relation is the reason why these components come to the fore of research 
on translation and interpreting teaching. Furthermore, it is described how all of the assumptions and represented theoretic concepts are applied in practice.

Keywords: postgraduate translator's education, strategic translation competence, curriculum of translator's education, didactic strategy, translation competence

Anna Małgorzewicz, Uniwersytet Wrocławski, Instytut Filologii Germańskiej, Pl. Nankiera 15b, 50-140 Wrocław, Polen, E-Mail: anna.malgorzewicz@uwr.edu.pl

Received: 30.09 .2018 , accepted: 8.04.2019

\section{Akademische Übersetzer- und Dolmetscherausbildung an polnischen Hochschulen}

Akademische Formen der Übersetzer- und Dolmetscherausbildung an polnischen Hochschulen werden in unterschiedlichen institutionellen und diversen paradigmatischen Kontexten der didaktisch-methodischen Gestaltung dargeboten. Ihre Repräsentationsformen bilden die translatorischen Studiengänge im Rahmen der Angewandten Linguistik, die translatorischen Spezialisierungen im Bereich der Neuphilologie, die auf der Fachbereichs-, Instituts- und Lehrstuhlebene vertreten sind, und die postgradualen Aufbaustudiengänge. Im Unterschied zu autonomen translatorischen Studiengängen werden die Inhalte der spezialisierungsbasierten Ausbildung sowie der Umfang des didaktischen Angebots vom wissenschaftlichen Profil der Ausbildungsstätte, den institutionellen, organisatorischen und personellen Modalitäten determiniert. Die Ersetzung der ministeriellen Standards durch den Nationalen Qualifikationsrahmen mit seiner Kompetenzzentriertheit sowie der Offenheit gegenüber neuen und vor allem aktuellen Ausbildungsbedürfnissen des Arbeitsmarktes hat sich zweifelsohne positiv auf die Schaffung von optimalen Lehrprogrammen ausgewirkt. Jedoch werden die zur Verfügung stehende geringe Anzahl der Leistungspunkte und somit der geringe Lehrstundenumfang sowie die philologisch verankerten Lerneffekte oft zu begrenzenden Determinanten bei der Gestaltung der Lehrprogramme. So müssen die Lernziele der translatorischen Spezialisierungen in philologischen Spezialisierungen jetzt mit den philologischen, auf der Ebene der Fachrichtung festgelegten Lernzielen kompatibel sein, welche wiederum direkten Bezug auf die humanistischen Lernziele nehmen. In diesem Sinne hat sich die Spezialisierungsform der Translationsdidaktik von ihrer philologischen Verankerung nicht befreit.

Im Fall der postgradualen Weiterbildungsformen verhält sich die Situation anders. Es besteht eine größere Flexibilität beim Formulieren der Lernziele, die dem Prinzip der Kompetenzzentriertheit verpflichtet ist. Obwohl das Interesse an der postgradualen Ausbildung in den letzten Jahren angesichts der Abschaffung der den Nicht-Philologen bzw. Nicht-Philologiestudenten auferlegten gesetzlichen 
Pflicht zur Absolvierung eines postgradualen Studiengangs als Voraussetzung zur Ablegung des Staatsexamens sowie aufgrund der polenweiten Institutionalisierung der Translatorenausbildung im Rahmen der philologisch verankerten Spezialisierungen nachgelassen hat (viele postgraduale Studiengänge wurden inzwischen abgeschafft), finden sich auch derzeit noch immer Kandidaten, die das Aufbaustudium für Translatoren am Institut für Germanistik der Universität Wrocław aufnehmen. Somit existieren heute bei uns beide Formen der Translatorenausbildung: die translatorische Spezialisierung im Magisterstudiengang und das postgraduale Aufbaustudium.

\subsection{Das Aufbaustudium für Translatoren am Institut für Germanistik der Universität Wrocław}

Die erste Edition des Studiums ist auf 2003 zu datieren. Die personellen Möglichkeiten des Instituts erlaubten es anfangs, im Rahmen des Studiums ein nur 208 Lehrstunden umfassendes Lehrprogramm zu realisieren, was natürlich lediglich eine Fokussierung auf die grundlegenden Probleme der dolmetscherischen und übersetzerischen Praxis gestattete. Das didaktische Hauptziel des Studiums war demzufolge anfangs die Ausbildung der translationsrelevanten Grundfähigkeiten und die Eröffnung von Perspektiven für die weitere Kompetenzentwicklung. Jedoch ermöglichten es die stetig wachsende Erfahrung, die gut entwickelten Partnerschaften mit entsprechenden Übersetzer- und Dolmetscherausbildungsstätten in Deutschland, wie dem Fachbereich Sprachen an der Hochschule Zittau/Görlitz und dem Fachbereich Angewandte Sprach- und Kulturwissenschaft an der Johannes-Gutenberg-Universität Mainz (Germersheim) sowie die Zusammenarbeit mit im außeruniversitären Bereich tätigen Übersetzern und Dolmetschern und der Polnischen Gesellschaft der Übersetzer für Wirtschaft, Rechts- und Gerichtswesen (TEPIS), das didaktische Angebot im Jahre 2006 auf 272 und im Jahre 2009 sogar auf 448 Lehrstunden zu erweitern (vgl. dazu Małgorzewicz 2004 und 2008). Damit konnte man u.a. den Erwartungen der Kandidaten entgegenkommen, die beabsichtigten, das Staatsexamen zum vereidigten Übersetzer anzugehen. Die Zahl der Bewerber überstieg die jährliche Aufnahmekapazität. Was dabei recht aufschlussreich scheint, war die Tatsache, dass die meisten Bewerber Germanisten mit einem Magisterabschluss waren. Gerade diese Gruppe erhoffte sich von dem postgradualen Studiengang die Ausbildung der translatorischen Teilkompetenzen. Und so bilden paradoxerweise nicht die Absolventen nichtphilologischer Fachrichtungen, denen das Gesetz 2005 die Pflicht zum Abschluss eines postgradualen Aufbaustudiums im Bereich der Sprachmittlung auferlegt hatte, das Gros der Studierenden, sondern eben Germanisten. Dieser Sachverhalt veranschaulichte damals auf eine sehr markante Weise die Diskrepanz zwischen den vom polnischen Ministerium für Hochschulbildung und Wissenschaft festgesetzten Bildungsstandards der neuphilologischen 
Studienrichtungen und den Standards für die Durchführung des Staatexamens. Eine analoge Erscheinung kann man auch heute beobachten. Obwohl der universitäre Abschluss einer translatorischen Ausbildung beim staatlichen Examen nicht mehr vorausgesetzt wird, sehen nicht nur die Absolventen der nicht-philologischen Studiengänge - also Kandidaten, die bisher keine Möglichkeit hatten, sich im Bereich der translationsrelevanten Tätigkeitsgebiete zu qualifizieren - die Notwendigkeit der translatorischen Weiterbildung, sondern auch Absolventen, welche bereits eine translatorische Spezialisierung absolviert haben. Grund dafür ist wahrscheinlich der größere Umfang der Lerninhalte und -ziele, welche im Rahmen der Spezialisierungen wegen institutioneller Begrenzungen nur ansatzweise behandelt werden können, dagegen im Lehrprogramm des postgradualen Studiums in den Fokus gezogen werden.

\subsubsection{Die kompetenzorientierte Programmkonzipierung}

Die mit dem Nationalen Qualifikationsrahmen eröffnete Möglichkeit der kompetenzorientierten Programmkonzipierung hat unseres Erachtens positive Konsequenzen für das Definieren der Lernziele, der Kompetenzbereiche und Evaluierungsformen gebracht. Weitere, das Planen der postgradualen Translationsdidaktik unterstützende Faktoren sind die größere Anzahl der Leistungspunkte im Vergleich zu den Spezialisierungen sowie die größeren finanziellen Spielräume, die die Einbeziehung professioneller Übersetzer und Dolmetscher sowie juristischer Fachleute in den didaktischen Prozess möglich machen.

\subsubsection{Empirische Untersuchungen im Bereich des translatorischen Wissens und der Translationsfähigkeiten}

Der Konzipierung des neuen, aktuell geltenden Lehrprogramms gingen Untersuchungen im Bereich des translatorischen Wissens und der Translationsfähigkeiten voraus, die in einer Gruppe von Germanistikstudenten und in einer Studentengruppe des postgradualen Studienganges am Institut für Germanistik durchgeführt wurden. Es handelte sich um eine Untersuchung, die die Ermittlung der individuellen Translationstheorien der Probanden (ein 23 Items umfassender Fragebogen zu Translationsdefinitionen, Translationsformen, Translatorrollen, translationsrelevanten Kompetenzbereichen, Eigenschaften des Translators, Determinanten der Translationstätigkeiten auf der Makro- und Mikroebene) und die Erforschung der kognitionsbasierten Übersetzungsprozesse mit der Verfolgung der Findungs- und Entscheidungswege zu Übersetzungen von Problemwörtern zum Ziel hatte. Die Triangulation der erhobenen Untersuchungsdaten (Fragebogendaten, Translat, Protokolle des LD) erlaubte folgende Defizite und Schwächen in den translationsrelevanten Fähigkeiten, Fertigkeiten, Haltungen und im translatorischen Wissen festzustellen (Małgorzewicz 2012): 
- rudimentäres autonomes, subjektzentriertes Verhalten und mangelndes strategisch-kommunikatives Handeln im Translationsprozess,

- geringe Selbstaufmerksamkeit, rudimentäres Kontroll- und (Selbst-)Regulationsverhalten,

- mangelnde Problemidentifikation (z.B. Nicht-Erkennen der „falschen Freunde" des Übersetzers, inkorrekte Lexemerkennung) und kaum entwickeltes strategisches Problemlösungsverfahren,

- zu geringes bzw. zu großes Selbstvertrauen,

- starke Wörterbuchorientierung; inadäquater und kritikloser Umgang mit Hilfsmitteln (volles Vertrauen in Wörterbuchdefinitionen, kaum entwickelte Selektier- und Revidierfähigkeit); kaum entwickelte Fähigkeit der Paralleltextnutzung beim Übersetzen, zu geringe Einbeziehung von Korpora der geschriebenen und gesprochenen Sprache, von Internetforen für Übersetzer, generelle Unkenntnis von translationsrelevanten Hilfsmitteln,

- fehlendes Gleichgewicht zwischen interner und externer kognitiver Unterstützung in der Verstehens- und Produktionsphase,

- syntagmatisches statt paradigmatisches und kontextgeleitetes Vorgehen bei der Bedeutungskonstruktion, kaum ausgebildetes eigenständiges Inferenzieren,

- wenig komplexe Handlungssequenzen bei der Lösungsfindung - kleine Anzahl von erwogenen tentativen Übersetzungsäquivalenten,

- wenig Freiraum für Kreativität, Assoziationsbildung,

- kein bzw. geringes Bewusstsein der translationsrelevanten Rolle des Recherchierers und des selbstagierenden Kommunikators,

- Beschränkung der Translatorrolle auf die Rolle eines Experten für Sprachen bzw. die Rolle eines „Wörterbuchs auf zwei Beinen“,

- Defizite im Bereich des metakognitiven und metasprachlichen Wissens.

\section{Ein strategisches Konzept der universitären Translationsdidaktik}

Die eruierten Defizite lieferten Indizien für die Formulierung der Lernergebnisse, die infolge der translatorischen Ausbildung erreicht werden sollen. Ohne an dieser Stelle detailliert auf die Untersuchungsergebnisse einzugehen (s. dazu Małgorzewicz 2012), lässt sich infolge der festgestellten Erscheinungen auf folgende Anforderungen (Postulate) hinweisen, die unseres Erachtens bei der Konzipierung der Lehrprogramme für die translatorische Ausbildung als maßgeblich gelten sollen:

- Bildung zum autonomen, entscheidungsbewussten und verantwortungsvollen Translator, 
- infolge der simultanen, kompatiblen und kohärenten Entwicklung in den Bereichen des translatorischen Wissens und der Translationsfähigkeiten und -fertigkeiten, die zusammen die Translatorkompetenz bilden, somit

- durch die Förderung der Entwicklung einer individuellen Translationstheorie als einer eigenen, der sich auf Grund der Translationspraxis und des reflektierten sowie theoriebasierten Umgangs mit den Translationserscheinungen bildenden die Vorstellung von der Translation in ihren vielfältigen Realisierungsformen, von den Translatorrollen und -aufgaben, der translationsrelevanten Verhaltens- und Handlungsmodi der Translatoren auf der Makro- und Mikroebene der Translatoraktivität,

- durch die Entwicklung von translationsrelevanten Haltungen, durch die Auseinandersetzung mit theoretischen Ansätzen und authentischen Translationssituationen,

- durch die Entwicklung von translationsrelevanten, entscheidungsund kommunikativ-strategiebasierten Verhaltens- und Handlungsformen im Bereich variabler kommunikationsorientierter Translationsformen,

- durch die Sensibilisierung für die Notwendigkeit der Weiterbildung im Berufsleben.

Die Ergebnisse der durchgeführten Untersuchungen haben die Notwendigkeit einer holistischen Ausbildung der translationsrelevanten Eigenschaften in Abhängigkeit von der jeweiligen Translationsform nachgewiesen. Gravierend nach unserer Auffassung von der Translatorkompetenz war ihr komplexer Aufbau, dessen Erfassung und Beschreibung in systematischer wie hierarchischer Hinsicht insofern schwer festzuhalten sind, als ihre Komponenten in einem wechselseitigen Dependenzverhältnis existieren und ihre Entwicklung dementsprechend nicht linear, sondern parallel verläuft. Man kann in Anlehnung an anerkannte Ansätze zwar annehmen, dass die Basis für die Herausbildung von automatisierten Operationen die Haltungen und Motivationen bilden (vgl. dazu L.M. Spencer/S.M. Spencer 1993; Małgorzewicz 2014), muss aber zugleich bedenken, dass diese auch einer ständigen Beeinflussung unterliegen und sich mit der wachsenden Erfahrung und ständigen Auseinandersetzung mit den Translationssituationen ändern. Wichtig für Translationsdidaktiker ist aber, dass solche Eigenschaften wie translationsrelevante Haltungen, Verhaltens- und Handlungsbereitschaften im didaktischen Prozess nicht nur durch eine gezielte Ausführung der Translationstätigkeiten gefördert werden, sondern auch zum Inhalt der Reflexion werden sollen.

\subsection{Die kommunikativ-strategische Translationskompetenz des Translators - das Hauptziel der translatorischen Ausbildung}

Es ist unverkennbar, dass die konzeptionelle Auseinandersetzung mit der Translationskompetenz unbedingt die Komponente ihrer kommunikativ-strategischen 
Ausrichtung berücksichtigen muss (vgl. dazu u.a. Hönig/Kußmaul 1982; Krings 1986; Żmudzki 1995, 2015; Małgorzewicz 2003, 2012, 2016a, 2016b). In dieser Hinsicht verstehen wir die Translationskompetenz als Befähigung zur handelnden, strategiegeleiteten Bewältigung komplexer Translationsaufgaben (in der Auffassung von Żmudzki 2013, 2015) im Rahmen diverser kommunikationsdeterminierter Translationsgefüge (vgl. Grucza 1981, 1993, 1998; Żmudzki 2013, 2015). Der Strategieeinsatz ist vom Translator - abgestimmt auf die Translationsaufgabe, auf den jeweiligen Ausgangs-, Zieltext und Zieltextadressaten - zu planen, zu überwachen und gegebenenfalls zu regulieren. Verschiedene Einsatzmodi der Strategien werden vom Translator in Abhängigkeit von den Translationsproblemen prospektiv entworfen, die zuvor sowohl auf der Mikro- als auch auf der Makroebene der Translationshandlung zu identifizieren sind. In der Fähigkeit der translationsspezifischen entscheidungsbasierten Problemlösung liegt unseres Erachtens der Kern der Translationskompetenz. ${ }^{1}$

Markant für die dargestellte Kompetenz-Auffassung ist die Fokussierung der Subjektzentriertheit. Im Falle der Translation äußert sich die subjektzentrierte Haltung im Verhältnis des Translators einerseits zu den Sprachen, die er spricht, und andererseits zu seiner Autonomität im Bereich der unternommenen Translationshandlung als Basis, auf der der Translator seine individuelle strategisch-kommunikative Translationskompetenz eigenständig entwickeln und einsetzen kann (Małgorzewicz 2014, 2015). Einen entscheidenden Stellenwert hat in dieser Sphäre das Identitätsbewusstsein des Translators.

Nicht nur die Erfüllung der genannten Anforderungen, sondern auch die Ausbildung entsprechender translationsrelevanter Fähig- und Fertigkeiten macht die Handlungen im Rahmen der Translationskommunikation möglich.

\subsection{Formulierung der Lernergebnisse - ein Faktorenmodell}

Den Ausgangspunkt bei der Konzipierung des Lehrprogramms war neben der Auflistung von diagnostizierten Defiziten im Bereich der Translationskompetenz der angehenden Translatoren auch die antizipatorische Bestimmung der auf die Formulierung der Lernergebnisse Einfluss nehmenden Faktoren. Diese konstitutiven Größen sind die reellen Translationssituationen (Translationsgefüge, charakterisiert durch konkret definierbare Komponenten: Translationsinitiator, AS-Textproduzent, AS-Text, Translator, ZS-Text, ZS-Adressat), Translationsformen, Sach(fach)gebiete, Rollen/Aufgaben des Translators, kommunikativ-strategisch bestimmte Hand-

${ }^{1}$ Vgl. dazu Weinerts Definition der Kompetenz, diese umfasst: „die bei Individuen verfügbaren oder durch sie erlernbaren kognitiven Fähigkeiten und Fertigkeiten, um bestimmte Probleme zu lösen, sowie die damit verbundenen motivationalen, volitionalen und sozialen Bereitschaften und Fähigkeiten, um die Problemlösungen in variablen Situationen erfolgreich und verantwortungsvoll nutzen zu können (Weinert 2001: 27 f.; vgl. dazu Małgorzewicz 2016a). 
lungen des Translators, Texte (mit ihrer Textsortenspezifik, Żmudzki 2015). Diese Faktoren und ihre Dependenzrelationen lassen sich schematisch wie folgt darstellen:

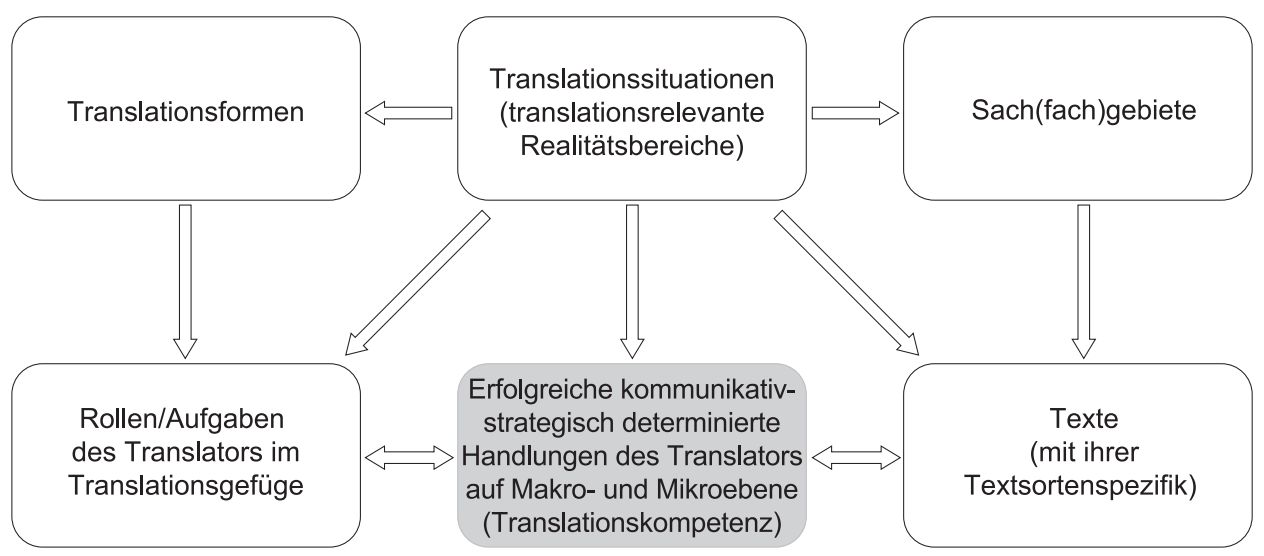

Abb.1: Konzipierung der Lehrprogramme - ein Faktorenmodell

Wie aus dem Schema ersichtlich ist, spielen beim Entwurf des translatorischen Lehrprogramms die Translationssituationen, mit denen der Absolvent potenziell konfrontiert wird, die ausschlaggebende Rolle für die Festlegung der Translationsformen, der Translatorrollen, der involvierten Sach(fach)gebiete sowie der in diesen Bereichen präsenten Textsorten und der translationsspezifischen Handlungen. Kommunikativ-strategisch determinierte Handlungen bilden das translationsdidaktische Hauptziel - das Lernergebnis -, dessen Erreichen die reflexive und interaktive Auseinandersetzung mit allen übrigen Komponenten in der Ausbildung voraussetzt.

Das Ziel unseres postgradualen Studiengangs ist die Ausbildung im Bereich der Vorbereitung zur Ausführung von Tätigkeiten des vereidigten Übersetzers/ Dolmetschers. Da dieser Beruf in Polen gesetzlich geregelt ist, sind auch seine Aktivitätsbereiche festgelegt. Wir bemühen uns, unsere Studierenden mit einer Vielfalt von möglichen Translationssituationen zu konfrontieren, auch mit solchen, die nicht unbedingt die Domäne des vereidigten Dolmetschers bzw. Übersetzers repräsentieren. Der in Polen vereidigte Translator muss - anders als in anderen europäischen Ländern - sowohl Dolmetsch- als auch Übersetzungstätigkeiten ausüben. Obwohl dieser Anforderung keine anerkannten und translationswissenschaftlich und -didaktisch begründbaren Prämissen zugrunde liegen, mussten wir beide Translationsformen im Lehrprogramm berücksichtigen. Neben dem Übersetzen wird daher auch die Dolmetschfertigkeit in ihren drei Varianten entwickelt: das Konsekutivdolmetschen ohne Notation, das Konsekutivdolmetschen mit Notation und das Blattdolmetschen. 
Das grundlegende Sachfachgebiet, mit dem wir unsere Studierenden konfrontieren, ist Jura. Diesem Sachfachgebiet wird das Modul Grundlagen des deutschen und polnischen Rechts gewidmet, das in Form einer inhaltsbezogenen Vorlesung und in Übungen realisiert wird. Ein weiteres stark repräsentiertes Sachfachgebiet ist u.a. Wirtschaft, diesem wie auch anderen Bereichen des Fachwissens, die in den behandelten Texten angesprochen werden, wird jedoch keine separate Veranstaltung gewidmet. Die ihnen zu Grunde liegenden Inhalte werden im Rahmen von Übungen behandelt.

Die Mehrheit der sich aus den relevanten Sachfachgebieten ergebenden Texte bilden schriftlich verfasste juristische, amtliche Schreiben, wie z.B. Gerichtsentscheidungen, Bescheide, Urteile, Vorladungen, Beschlüsse, Testamente, gerichtliche und polizeiliche Protokolle, notarielle Beurkundungen, Verträge, Buchführungsdokumente (Jahresabschlussberichte, Lageberichte, Bilanzen, Gewinn- und Verlustrechnungen), Handelsdokumente (Rechnungen, Kassenzettel) und -korrespondenz, Steuerdokumente (Finanzamtbescheinigungen, Lohnsteuerkarten, Einkommen(s)steuererklärungen (PIT-Formular)). Eine gesonderte Gruppe bilden Dokumente, wie z.B. Urkunden, Zeugnisse, Arbeitszeugnisse, Referenzschreiben, Dokumente bezüglich der Anerkennung und des Abstreitens der Vaterschaft eines Kindes. Eine weitere Gruppe der didaktisierten Texte bilden die Gebrauchstexte: Kochrezepte, Gebrauchsanleitungen, Tourismusprospekte, Geschäftsordnungen, Werbematerial, Packungsbeilagen für Arzneimittel, Bestellungen. Im Fall der Dolmetschsituationen handelt es sich bspw. um folgende Texte: Rede, Vortrag, Interview, Belehrung eines Beschuldigten, Verhör eines Beschuldigten, Haftbefehl, Anklage sowie die bereits genannten, primär schriftlichen Textsorten, die in Dolmetschsituationen vom Blatt gedolmetscht werden.

Die letzte Komponente in unserer Aufstellung curricularer Einflussgrößen sind die Translatorrollen, die im Translationsprozess ausgeführt werden. Der Translator ist in erster Linie der Ausführende von kognitiv gesteuerten Übersetzungs- und Dolmetschtätigkeiten in der Translationskommunikation. Berücksichtigt werden in unserem Lehrprogramm aber auch andere translationsrelevante Funktionen; wie z.B. die des Terminologen, des Recherchierers, des Korrektors, des Posteditors, des Beraters, des Helfers bei Verständnisproblemen. Die genannten Rollen werden in aufgabenorientierten praktischen Lehrveranstaltungen gezielt an die Studierenden angepasst.

\subsection{Lehrveranstaltungen und ihre Inhalte}

Die Translationssituationen, in denen die konkretisierten Textsorten als Kommunikationsinstrumente eingesetzt werden und in denen unsere Studierenden bestimmte translationsrelevante Rollen ausführen sollen, werden zum Unterrichtsinhalt praxisorientierter Lehrveranstaltungen. Es handelt sich hierbei um Übungen 
im Modul Übersetzen: Übersetzen von Gebrauchstexten, Übersetzen von Dokumenten, Übersetzen von juristischen Texten und Übersetzen von Finanztexten sowie Übungen im Modul Dolmetschen und Notationstechnik. Der Lehrprozess setzt mit dem Übersetzen von Gebrauchstexten ein. In dieser funktional angelegten Lehrveranstaltung wird großer Wert auf das Bewusstmachen der kommunikativen Verankerung der Translation gelegt. Neben den Translationsaufgaben (immer mit vollständiger Angabe aller die Translationskommunikation bildenden Konstituenten) wird die translationsorientierte Textanalyse in ihrer kommunikativen/diskursiven Einbettung durchgeführt. Ein wichtiges Element des Unterrichts sind überdies Dokumentationen, Protokolle bzw. Kommentare zu den angefertigten Übersetzungen. Sie bilden die Basis für Diskussionen, für das Diagnostizieren der begangenen Übersetzungsfehler sowie die Grundlage für die Übersetzungsbewertung. In dieser Veranstaltung werden unterschiedliche Sozialformen eingesetzt: Einzelübersetzen, Zu-Zweit-Übersetzen und kooperatives Übersetzen. Die Vielfalt der behandelten Translationssituationen ermöglicht die Wahrnehmung der divergenten Translatorrollen durch die Studierenden. Die Lehrveranstaltung Übersetzen von Gebrauchstexten soll eine Art Propädeutik für alle anderen Lehrveranstaltungen im Übersetzen schaffen.

Basalen Charakter für die Ausbildung der übersetzens- und dolmetschrelevanten Handlungs- und Verhaltensformen haben auch die im ersten Semester angebotenen Vorlesungen Grundlagen der Translatorik, rechtlicher Status sowie Berufsethik des Übersetzers/Dolmetschers. Diese Lehrveranstaltungen ermöglichen den Studierenden einerseits die theoriegestützte Auseinandersetzung mit anerkannten Modellen diverser Translationsformen und der translationswissenschaftlichen Beschreibung der sie konstituierenden Phänomene und andererseits den Erwerb und die Vertiefung von Kenntnissen im Bereich der beruflichen, gesetzlich und im Kodex des vereidigten Übersetzers/Dolmetschers geregelten Tätigkeit.

Eine unterstützende Rolle bei der Entwicklung der Translationsfähigkeiten und -fertigkeiten erfüllen Lehrveranstaltungen, welche das metasprachliche Bewusstsein der Studenten auf ein höheres Niveau bringen. Es handelt sich um Übungen zur Stilistik der polnischen Sprache und Übungen zur Stilistik der deutschen Sprache sowie um das Seminar Elemente der deutsch-polnischen kontrastiven Grammatik und das Seminar Künstlerisches Übersetzen.

Ein fester Bestandteil unseres Curriculums ist die Ausbildung der IT-Fertigkeiten. In einem gesonderten Kurs werden die Studierenden mit Translation Memory Systems (TMS) vertraut gemacht. Sie haben die Möglichkeit, sich in eines von diesen Systemen - TRADOS - einzuarbeiten.

Die vorgenommene Darstellung der Schwerpunkte einzelner Lehrveranstaltungen gibt Einsicht in ihre Vernetzung und ihre gegenseitige Bedingtheit. Die festgelegte Progression der Lehrinhalte, die Entwicklung der Translationskompetenz im aufgabenorientierten Lehrprozess soll das Erreichen der Lernergebnisse sichern. Die integrative Entwicklung der für die translatorische Ausbildung defi- 
nierten Wissens-, Fähig- und Fertigkeitsbereiche soll im Endeffekt zur Entfaltung der strategisch-kommunikativen Translationskompetenz beitragen, die dem angehenden Translator das autonome, reflexive, entscheidungsbasierte, verantwortungsvolle Handeln in variablen Realitätsbereichen der Translationswirklichkeit und unter Einsatz von den kommunikativen Translationszielen adäquaten Zieltexten ermöglicht.

\subsection{Lernergebnisse und Leistungsmessung}

So wie der Qualifikationsrahmen dies verlangt, haben wir für jedes Modul Bereiche des Wissens, der Lernergebnisse und der s.g. sozialen Kompetenzen definiert. Aufgrund der spezifizierten curricularen Einflussgrößen konnten die für Translationshandlungen konstitutiven Fähig- und Fertigkeiten definiert werden. So sind detaillierte Kataloge von Handlungs- und Verhaltensformen, ergänzt durch eine Auflistung von translationsrelevanten Kenntnissen, entstanden. Da das Niveau der Translationskompetenz sich an der Qualität der ausgeführten Handlungen ablesen lässt, sollen die Lernergebnisse mittels adäquater Verfahren überprüft werden. Daher beurteilen wir im Rahmen der einzelnen Fächer die kommunikativ-strategisch determinierten Handlungen der angehenden Translatoren auf Grund der von ihnen erbrachten Translationsleistungen in komplexen, realistischen oder auch authentischen Translationssitationen. Es wird dabei darauf geachtet, dass den Studierenden alle möglichen Hilfsmittel zur Verfügung stehen. Auf diese Weise soll nicht nur die Qualität der Translationsoperationen bewertet werden, sondern auch die Recherchierleistung (auch im Fall der Dolmetschfertigkeit), die Kenntnis von Internet-Glossaren und Terminologie-Datenbanken sowie die Fähigkeit, mit Paralleltexten zu arbeiten. $\mathrm{Zu}$ beurteilen ist auch die sprachliche Gewandtheit, im Besonderen beim Dolmetschen, das den Einsatz bestimmter Dolmetschtstrategien erfordert und somit völlig andere Bewertungskriterien verlangt als das Übersetzen. Bei der Beurteilung der Dolmetschfertigkeit soll auch auf die erfolgreiche Anwendung der Notationstechnik Bezug genommen werden.

\subsubsection{Eingesetzte Prinzipien zur Beurteilung der Translationsleistung vs. Voraussetzungen der staatlichen Prüfung zum vereidigten Dolmetscher/Übersetzer}

Die angeführten Prinzipien zur Beurteilung der Translationsleistung decken sich leider nicht mit den Voraussetzungen der staatlichen Prüfung zum vereidigten Dolmetscher/Übersetzer, die unseres Erachtens nicht kompetenzorientiert angelegt sind. Kontrovers sind auch das Prüfungsformat, die verwendeten Prüfungstexte, die Durchführungsbestimmungen für die einzelnen Prüfungsteile sowie die Be- 
wertungskriterien. Sie stehen nicht im Einklang mit den Prüfungsformen, die dem handlungsorientierten Konzept der Bologna-Reform entsprechen würden. Da wir unseren Aufbaustudiengang auch als Vorbereitung auf die staatliche Prüfung anbieten, verwenden wir deren Format bei der Abschlussprüfung. Wir wollen damit den Studierenden die Möglichkeit schaffen, sich mit der zu erwartenden Prüfungssituation vertraut zu machen. Es bleibt zu wünschen, dass die staatliche Kommission die Prüfungskriterien mit den Erkenntnissen der neuesten Forschung zur Leistungsmessung in Einklang bringt, wobei auch auf bewährte Prüfungsformate aus anderen europäischen Ländern zurückgegriffen werden könnte.

\section{Die fachbezogene Translationsdidaktik - Für und Wider}

Unsere Erfahrungen zeigen, dass die Ausbildung der Translationskompetenz ein sehr komplexer Prozess ist. Mit der Integrierung des fachbezogenen Wissens, das im Fall des vereidigten Übersetzers/Dolmetschers von Relevanz ist, haben wir das von uns vertretene Konzept der Translationsdidaktik in die Bahnen einer fachbezogenen Spezialisierung für Übersetzer/Dolmetscher gelenkt. Es stellt sich aber die Frage, ob der Anteil des deklarativen Fachwissens im Lehrprogramm immer so hoch sein muss. Das Ziel der Translationsdidaktik besteht unseres Erachtens eher in der Förderung und Stärkung der genannten Haltungen, und nicht unbedingt in der Vermittlung einer bestimmten Menge von Fachwissen, zumal man nicht voraussehen kann, mit welchen Fachgebieten die Absolventen in ihrer beruflichen Tätigkeit tatsächlich in Berührung kommen werden.

\section{Literatur}

Grucza, Franciszek (1981): Zagadnienia translatoryki. In: Grucza, Franciszek (Hrsg.): Glottodydaktyka a translatoryka. Warszawa. S. 9-29.

Grucza, Franciszek (1993): Interkulturelle Translationskompetenz: ihre Struktur und Natur. In: Frank, Armin P. / Maaß, Kurt-Jürgen / Paul, Fritz / Turk, Horst (Hrsg.): Übersetzen, verstehen, Brücken bauen. Geisteswissenschaftliches und literarisches Übersetzen im internationalen Kulturaustausch. Berlin. S. 158-171.

Grucza, Franciszek (1998): Wyodrębnienie się, stan aktualny i perspektywy świata translacji oraz translatoryki. In: Lingua Legis 6, S. 2-12.

Hönig, Hans G. / Kußmaul, Paul (1982): Strategie der Übersetzung: ein Lehr- und Arbeitsbuch. Tübingen.

Krings, Hans P. (1986): Was in den Köpfen von Übersetzern vorgeht. Eine empirische Untersuchung zur Struktur des Übersetzungsprozesses an fortgeschrittenen Französischlernern. Tübingen.

Małgorzewicz, Anna (2003): Prozessorientierte Dolmetschdidaktik. Wrocław. 
Małgorzewicz, Anna (2004): Entwicklung der translatorischen Kompetenz im Rahmen des Postgradualen Studiums für Sprachmittler an der Universität Wroclaw. In: Fifth International Symposium on Teaching Translation and Interpreting: BA/MA Programmes - an internationale perspective, Germersheim, 9-11 December 2004, www.fask.uni-mainz.de/user/hagemann/ publ/malgorzewicz.pdf.

Małgorzewicz, Anna (2008): Entwicklung der translatorischen Kompetenz im Rahmen des Postgradualen Studiums für Übersetzer und Dolmetscher am Institut für Germanistik an der Universität Wrocław. In: Glottodidactica 34, S. 53-63.

Małgorzewicz, Anna (2012): Die Kompetenzen des Translators aus kognitiver und translationsdidaktischer Sicht. Wrocław.

Małgorzewicz, Anna (2014): Językowe i niejęzykowe kompetencje tłumacza. Próba zdefiniowania celów translodydaktyki akademickiej. In: Lingwistyka Stosowana/Applied Linguistics/Angewandte Linguistik 11, S. 1-10.

Małgorzewicz, Anna (2016a): Translationsdidaktik in Polen. Aktueller Stand und Perspektiven. In: Żebrowska, Ewa / Olpińska-Szkiełko, Magdalena / Latkowska, Magdalena (Hrsg.): Zwischen Kontinuität und Modernität: Metawissenschaftliche und wissenschaftliche Erkenntnisse der germanistischen Forschung in Polen. Warszawa. S. 107-116.

Małgorzewicz, Anna (2016b): Podejście zadaniowe w antropocentrycznej translodydaktyce akademickiej. In: Lingwistyka Stosowana/Applied Linguistics/Angewandte Linguistik 19, S. 149-165.

Spencer, Lyle M. / Spencer Signe M. (1993): Competence at Work. Models for Superior Performance. New York.

Weinert, Franz E. (2001): Vergleichende Leistungsmessung in Schulen - eine umstrittene Selbstverständlichkeit. In: Weinert, Franz E. (Hrsg.): Leistungsmessung in Schulen. Weinheim. S. 17-32.

Żmudzki, Jerzy (2013): Holizm funkcjonalny w perspektywie translatoryki antropocentrycznej. In: Lingwistyka Stosowana/Applied Linguistics/Angewandte Linguistik 8, S. 177-187.

Żmudzki, Jerzy (2015): Blattdolmetschen in paradigmatischer Perspektive der anthropozentrischen Translatorik. Frankfurt am Main. 